\title{
Perceptions Paysannes Des Aléas Climatiques Sur La Production Du Riz Sur Le Périmètre Irrigué De Saga Dans La Vallée Du Fleuve Niger
}

\author{
Seydou Tahirou Moussa
}

Université Aube Nouvelle, Unité de Formation et de Recherche en Sciences et Technologies (UFR/ST), Laboratoire de Recherche et d'Etudes en Management (CREM) Ouagadougou, / Burkina Faso

\section{Patrice Zerbo}

Université Joseph KI-ZERBO, Unité de Formation et de Recherche en Sciences de la Vie et de la Terre (UFR/SVT), Laboratoire de Biologie et Écologie Végétales, Ouagadougou, Burkina Faso

\section{Yacouba Sanou}

Samuel Paré

Université Joseph KI-ZERBO, Unité de Formation et de Recherche en

Sciences Exactes et Appliquées (UFR/SEA),

Laboratoire de Chimie Analytique, Environnementale et Bio-Organique, Ouagadougou / Burkina Faso

\section{Manan Nassirou Ado}

Université de Tahoua, (UFR/FSA),

Faculté des Sciences Agronomiques, Tahoua/Niger

Doi:10.19044/esj.2022.v18n3p217

Submitted: 09 November 2021

Accepted: 19 January 2022

Published: 31 January 2022
Copyright 2022 Author(s)

Under Creative Commons BY-NC-ND 4.0 OPEN ACCESS

Cite As:

Tahirou Moussa S., Zerbo P., Sanou Y., Pare S., \& Nassirou Ado M.,(2021). Perceptions Paysannes Des Aléas Climatiques Sur La Production Du Riz Sur Le Périmètre Irrigué De Saga Dans La Vallée Du Fleuve Niger European Scientific Journal, ESJ, 18 (3), 217.

https://doi.org/10.19044/esj.2022.v18n3p217

\section{Résumé}

L'objectif général poursuivi est d'analyser la perception des aléas climatiques auprès des exploitants en milieu urbain. Pour ce faire, des entretiens semi-structurés et des enquêtes ont été effectués. Au total 140 exploitants disponible ont été enquêtés. Un critère d'âge de sélection des exploitants variant de 15 à 50 ans a permis de s'assurer que les stratégies développées font suite à une perception effective des changements. L'analyse 
des données a été faite par le logiciel Access et Excel pour le calcul des paramètres statistiques descriptifs et la réalisation de graphiques. Ces derniers observent l'existence des paramètres climatiques qui interviennent dans leur pratique à 90\%. Les résultats de la recherche révèlent que $66 \%$ des exploitants ont un niveau d'instruction assez bas. Face aux aléas climatiques les exploitants mettent en œuvre des stratégies durables d'adaptations qui participent à l'amélioration des rendements. $80 \%$ des exploitants font recourt aux variétés locales et adoptent des stratégies diverses à la pratique d'irrigation et Le drainage et le curage des cours d'eau (30\%) ;adoption des variétés résistantes au stress thermique et de variétés de cycle court (65\%), décalage des dates de semis et irrigation (15\%).Les principaux déterminants de l'adoption des stratégies adaptatives sont variables de perception. De ce fait, la nature et l'efficacité des stratégies utilisées mettent en évidence le caractère vulnérable des exploitants par des contraintes matérielles, financières et techniques et l'accès à l'information. Ainsi, malgré les stratégies mises en place, les exploitants sont soumis d'une part à une vulnérabilité tant sociale qu'aux vulnérabilités de production.

Mots clés: Changement climatique, Perception, Adaptation, vulnérabilité, riziculture urbaine 


\title{
Farmers' Perceptions of Climatic Hazards on Rice Production in the Irrigated Area of Saga in the Niger River Valley
}

\section{Seydou Tahirou Moussa}

Université Aube Nouvelle, Unité de Formation et de Recherche en Sciences et Technologies (UFR/ST), Laboratoire de Recherche et d'Etudes en Management (CREM) Ouagadougou, / Burkina Faso

\section{Patrice Zerbo}

Université Joseph KI-ZERBO, Unité de Formation et de Recherche en Sciences de la Vie et de la Terre (UFR/SVT), Laboratoire de Biologie et Écologie Végétales, Ouagadougou, Burkina Faso

\section{Yacouba Sanou}

Samuel Paré

Université Joseph KI-ZERBO, Unité de Formation et de Recherche en Sciences Exactes et Appliquées (UFR/SEA),

Laboratoire de Chimie Analytique, Environnementale et Bio-Organique, Ouagadougou / Burkina Faso

\section{Manan Nassirou Ado}

Université de Tahoua, (UFR/FSA),

Faculté des Sciences Agronomiques, Tahoua/Niger

\begin{abstract}
The general objective is to analyze the perception of climatic hazards among farmers in urban areas. To do this, semi-structured interviews and surveys were conducted. A total of 140 available farmers were surveyed. An age criterion for the selection of farmers ranging from 15 to 50 years old was used to ensure that the strategies developed were based on an effective perception of changes. The data was analyzed using Access and Excel software to calculate descriptive statistical parameters and to produce graphs. The graphs show the existence of climatic parameters that are involved in $90 \%$ of their practices. The results of the research reveal that $66 \%$ of the farmers have a fairly low level of education. Faced with climatic hazards, farmers implement sustainable adaptation strategies that help improve yields. $80 \%$ of farmers use local varieties and adopt various strategies for irrigation and drainage (30\%); adoption of heat stress resistant varieties and short cycle varieties (65\%); and shifting of sowing dates and irrigation (15\%). The main determinants of the adoption of adaptive strategies vary in perception. As a result, the nature and effectiveness of the strategies used highlight the vulnerability of farmers due to material, financial and technical constraints and access to information. Thus, despite the strategies implemented, farmers are
\end{abstract}


subject to both social and production vulnerabilities.

Keywords: Climate change, Perception, Adaptation, Vulnerability, Urban rice production

\section{Introduction}

Le changement climatique apparaît de nos jours comme la menace la plus préoccupante à la réalisation des aspirations de réduction de la pauvreté des pays africains et constitue de ce fait un frein à l'atteinte des Objectifs du Millénaire pour le Développement (OMD). Il affecte la pluviométrie, la disponibilité de l'eau, le niveau des mers, l'augmentation des sécheresses et la fréquence des feux de brousse. De plus en plus, son impact sur la santé humaine, la productivité agricole et la biodiversité est très visible particulièrement en Afrique. En effet, l'imprévisibilité de la croissance de ce changement climatique constitue une menace pour le secteur agricole et un frein pour l'atteinte de l'autosuffisance alimentaire (Ali et Erenstein, 2017). Les effets potentiels du changement climatique sur la productivité agricole suscitent de nombreuses préoccupations (Gomgnimbou et al.,2020), De ce fait, la plupart des pays d'Afrique de l'Ouest particulièrement les pays sahéliens à saisons alternées, sont très vulnérables aux changements climatiques (Bonkoungou et al., 2019), ce qui fragilise les systèmes agricoles qui ne répondent plus aux pressions actuelles du climat (Yegbemey et al.2014). Ce changement est perçu par des agriculteurs à travers divers évènements susceptibles de produire des effets négatifs sur les exploitations (Harvey et al., 2014 ; Kasongo et Mosombo, 2017). Cette menace des aléas climatiques suscite chez les producteurs une incertitude et une perte des repères saisonniers (Pierre et al. 2012 ; Donkora, 2019). Plusieurs études ont été réalisées sur l'impact du changement climatique, notamment sur la perception des communautés locales et sur les options d'adaptation utilisées (Saboury, 2018). Les riziculteurs perçoivent les effets du changement climatique et développent plusieurs stratégies d'adaptation (Abdou et al., 2014) de manière endogène pour réduire les effets néfastes de ces variabilités climatiques sur la production. Les travaux recensés sur les stratégies d'adaptation indiquent que l'utilisation des semences résistantes à la sècheresse associée à l'agroforesterie, le décalage des dates de semis et l'irrigation (Sarr et al., 2015), la gestion efficiente de l'eau et des fertilisants ainsi que la diversification des cultures sont parmi les options d'adaptation privilégiées (Bele et al., 2014 ; Makate et al., 2016). Cependant, la mise en place de ces stratégies varie d'un système de production à l'autre, des opportunités, des facteurs socio-économiques ainsi que de la perception des agriculteurs du changement climatique (Abid et al., 2015 ; Asayehegn et al., 2017). Ainsi, la présente recherche, tente de savoir de quelles manières ces 
exploitants parviennent-elles à faire face aux défis du changement climatique ? L'objectif de cette recherche est d'analyser la perception paysanne des aléas climatiques sur la riziculture dans la vallée du fleuve Niger.

\section{Matériel et méthodes}

\subsection{Présentation de la zone de de recherche}

La présente recherche s'est réalisée sur le périmètre irrigué de Saga situé entre $\left(13^{\circ} 27^{\prime} 39,9^{\prime}\right.$ 'N $, 002^{\circ} 08^{\prime} 44,1^{\prime}$ 'E) dans la Commune IV de la ville de Niamey au Niger (Figure 1). Le site est sous l'influence d'un climat sahélosoudanien qui se traduit par l'alternance de deux saisons distinctes : une saison sèche qui s'étend du mois d'octobre à mai et, une saison pluvieuse qui s'étend de juin à septembre avec une température moyenne de 28 à $42^{\circ} \mathrm{C}$ (Ozer, 2017). Les sols sont riches en sédiments d'origine alluviale avec une texture argileuse. D'après les résultats des travaux de recherche de Issiaka et al. (2018), le couvert végétal est essentiellement constitué d'espèces locales telles que : Acacia nilotica, Bauhinia refescens, Pourparcia birrea, Delonix regia, Adansonia digitata (baobab), Khaya sengalensis (caïcédrat), Acacia seyal, Balanites aegyptiaca, Ceiba pentandra, Prosopis africana, Ziziphus mauritania, Cassia sieberiane, Ficus, Hyphaene thebaica (doum) et Parkia biglobosa (Néré). Les espèces exotiques, plantées, sont essentiellement le neem (Azadirachta indica), l'eucalyptus (Eucalyptus camaldulensis), le mélina (Gmelina arborea), Terminalia mentaly, Prosopis juliflora, Cassia siamea, Acacia holocericea. Le réseau hydrographique est constitué par un seul cours d'eau permanant le fleuve Niger (Bahari et al., 2018). 


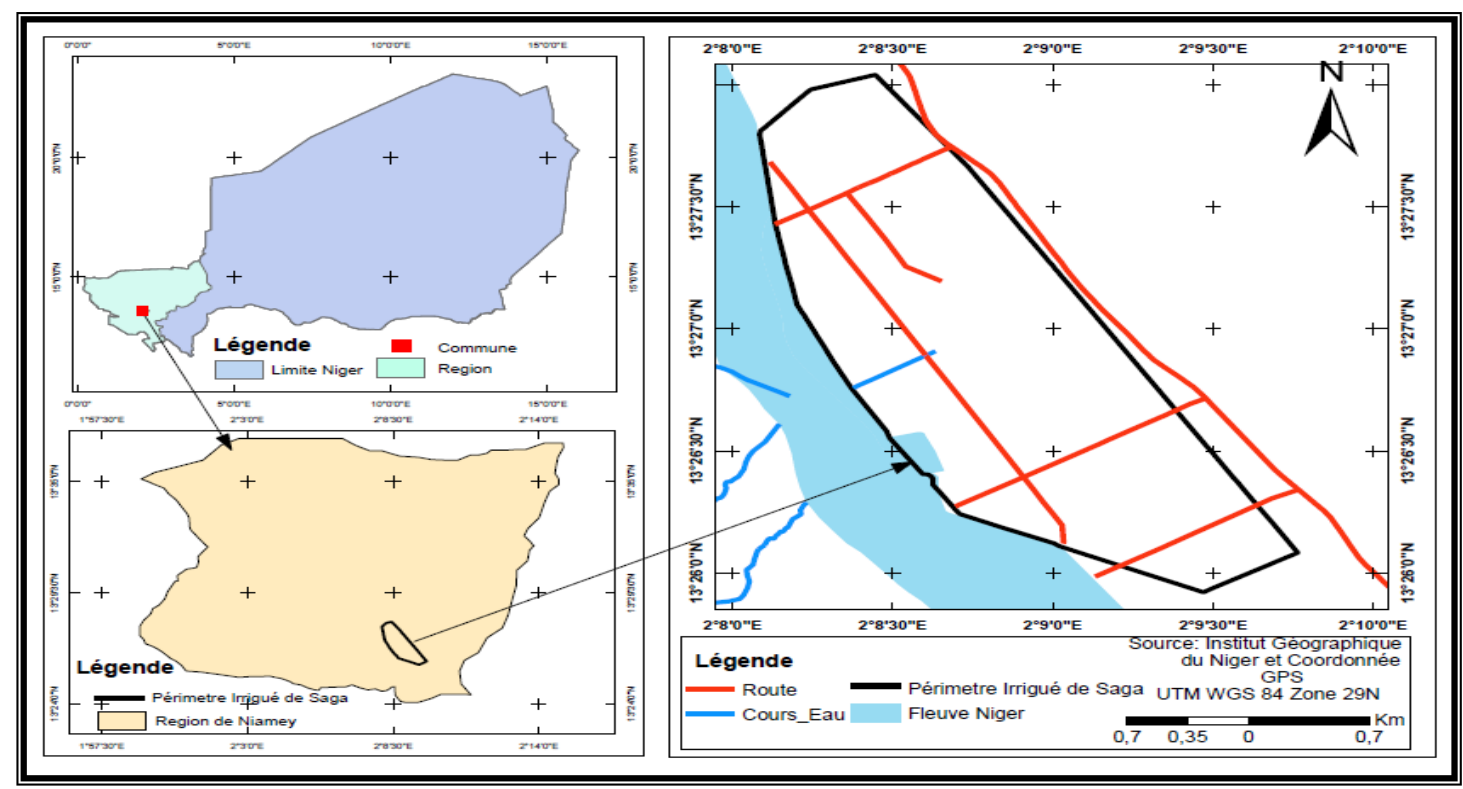

Figure 1 : Cartographie du périmètre irrigué de saga

Source : Institut Géographique du Niger (2021)

La figure 1 ci-dessus illustre l'aménagement hydro-agricole de Saga et sa répartition en Groupement Mutualiste de Production(GMP). Le périmètre irrigué est aménagé depuis 1987 pour la riziculture intensive sur une superficie nominale de 431 ha dont 381 ha dominées par le réseau d'irrigation et 49 ha pour le maraichage. La culture du riz est pratiquée selon un calendrier cultural de deux campagnes par an : une première campagne saison sèche (SS) qui va de Novembre à Mai et une campagne de saison d'hivernage $(\mathrm{SH})$ qui va de Juin jusqu'en décembre.

\subsection{Méthodes}

\subsubsection{Echantillonnage}

Les unités d'observation sont les producteurs du riz. Dans chaque Groupement Mutualiste de Production (GMP) au nombre (7) sélectionnés, 20 exploitants ont été constitués de manière aléatoire à choix raisonné. Ainsi, au total 140 exploitants disponible ont été enquêtés (Tableau 1). Un critère d'âge de sélection des exploitants variant de 15 à 50 ans a permis de s'assurer que les stratégies développées font suite à une perception effective des changements. Les données ont été collectées par des enquêtes à travers des entretiens semi structurés. Elles sont qualitatives et quantitatives. Elles ont été collectées durant la campagne agricole 2020-2021. Les travaux de Kasongo et Mosombo (2017) et Kabore et al. (2019) nous ont orientés du point de vue méthodologique pour la structuration du questionnaire. Ces données sont relatives(i) aux caractéristiques sociodémographiques des exploitants, (ii) les 
paramètres d'appréciation du changement climatique, (iii) perceptions sur les variétés cultivées et la double riziculture, (iv) les stratégies d'adaptations adoptées. Le dialogue est fait en langue locale (Zarma et Haoussa) dans le cas où certains producteurs ne comprennent pas la langue française ce qui a permis de recueillir les informations communiquées.

\subsubsection{Analyses des données}

Deux (2) types d'analyse ont été effectués. Il s'agit de l'analyse quantitative et l'analyse qualitative. L'analyse quantitative a permis le regroupement des données issues des questionnaires à l'aide de l'outil informatique tels qu'Access 2013 pour la saisie des données collectées afin de constituer une base de données, puis Excel 2013 pour agréger le calcul des paramètres statistiques descriptifs.

Quant à l'analyse qualitative, elle renvoie l'analyses des données relatives à la perception du climat sur la base d'une analyse en composantes multiples :

- Une perception du climat est qualifiée de «bonne », si les réponses de l'enquêté sur le climat recoupaient les observations réalisées au cours des dix dernières années (Kosmowski et al., 2015). Si la description du climat était juste pour au moins sept des huit critères suivants (Augmentation des pluies ; saison des pluies ; démarrage tardif des pluies ; fin des pluies tardive ; variation forte des pluies d'une année sur l'autre ; augmentation de la température maximale ; augmentation de la température minimale), il est considéré que l'exploitant a une bonne perception du climat (Yegbemey et al., 2014).

- Les stratégies locales d'adaptation ont été identifiées par le choix variétal de riz et les stratégies endogènes de gestion des aléas climatiques ;

- La détermination des facteurs de vulnérabilité basée sur la vulnérabilité des exploitants est liée d'une part aux activités de production et d'autre part aux ressources en capital humain, financier, social et physique qui déterminent les capacités de réaction des personnes face aux différents chocs (Bonkoungou et al., 2019).

- Les deux critères d'efficacité et limites des stratégies d'adaptations retenus renvoient ainsi à la fois à des questions de viabilité et diversification

- Si les stratégies peuvent engendrent des effets immédiats et à court terme, elle est considérée faible

- Si les stratégies peuvent engendrent des effets à long terme, elle est considéré efficace 


\section{Résultats}

\subsection{Caractéristiques sociodémographiques des exploitants du site de} recherche

Les caractéristiques liées aux exploitants (Tableau 1) sont essentiellement de quatre (4) ordres : l'âge, le sexe, le niveau d'instruction et l'expérience acquise des riziculteurs.

Tableau 1 : Répartition des riziculteurs selon leurs caractéristiques sociodémographiques

\begin{tabular}{|c|c|c|}
\hline Caractéristique des riziculteurs & Effectifs & Pourcentage $(\%)$ \\
\hline \multicolumn{3}{|l|}{ Age (ans) } \\
\hline$[15-30[$ & 36 & 26 \\
\hline$[30-40[$ & 61 & 44 \\
\hline$[40-50[$ & 43 & 30 \\
\hline \multicolumn{3}{|l|}{ Sexe } \\
\hline Hommes & 96 & 69 \\
\hline Femmes & 44 & 31 \\
\hline \multicolumn{3}{|l|}{ Niveau d'instruction } \\
\hline Non alphabétisés & 92 & 66 \\
\hline Primaire & 25 & 18 \\
\hline Alphabétisé & 13 & 9 \\
\hline Supérieur & 10 & 7 \\
\hline \multicolumn{3}{|l|}{ Expérience acquise } \\
\hline$\geq 10$ & 66 & 47 \\
\hline$\leq 10$ & 74 & 53 \\
\hline
\end{tabular}

L'analyse des données du tableau 1 montre que les exploitants sont composés essentiellement des hommes qui représentent $96 \%$ et $44 \%$ des femmes de l'effectif de l'échantillon. L'âge des enquêtés varie de 15 à 50 ans, avec la moyenne d'âge se situant autour de 30 ans. Sur l'ensemble des enquêtés, les exploitants âgés moins de 30 ans représentent $26 \%$, ceux âgés de 30 à 40 ans $44 \%$ et ceux de plus de 40 ans représentent $30 \%$. Les exploitants dans leur ensemble sont non alphabétisés à $66 \%$ et ceux ayant été à l'école présentent $34 \%$, dont $18 \%$ ont suivi l'école primaire, $9 \%$ ont atteint le cycle secondaire et $7 \%$ le cycle supérieur Concernant leur niveau de l'expérience acquise, 74 $\%$ des exploitants totalisent 10 à 20 ans dans la pratique rizicole.

\subsection{Perception du changement climatique selon les exploitants}

Les résultats révèlent que $90 \%$ des exploitants confirment l'existence de paramètres climatiques observables au niveau de la pluviométrie, de la température, du vent et des inondations. Le tableau2 consigne l'état de la perception climatique par les exploitants. 
Tableau 2 Evolution des facteurs climatiques selon les enquêtés.

\begin{tabular}{cccc}
\hline Facteurs & Augmenter & Stable & Taux (\%) \\
\cline { 1 - 2 } Pluviométrie & +++ & & $90 \%$ \\
\cline { 1 - 3 } Température & +++ & ++ & $60 \%$ \\
\hline Vent & & ++ & $80 \%$ \\
\hline Inondations & & +1
\end{tabular}

Source : Résultats de l'enquête, 2021

+++ : Augmente ; ++ : Stable

La lecture du tableau 2 montre que le changement climatique est perçu de diverses manières par les exploitants. Un fort taux des enquêtés (80 \%) estiment que ces principaux aléas climatiques à un effet négatif sur la riziculture irriguée d'une manière ou d'une autre et perturbent l'équilibre général des rendements rizicoles.

\subsection{Stratégies locales d'adaptations}

Face à la dégradation continue de leurs conditions de vie dans le contexte actuel de variabilité climatique, les exploitants ont développé diverses stratégies d'adaptation notamment le choix variétal et celui des systèmes de cultures.

\subsubsection{Variétés du riz utilisées par les exploitants}

Les résultats des enquêtes ont révélé que les exploitants font recours à cinq variétés de riz dont deux homologuées (Gambiaka; IR15-29) et trois locales (Waihidjo ; $\mathrm{N}^{\circ} 1 ; \mathrm{N}^{\circ} 2$ ). Cependant, $80 \%$ (Tableau 3) des exploitants utilisent les variétés locales dans les stratégies d'adaptation. Le tableau 3 présente les différents pourcentages des assertions d'enquêtés.

Tableau 3 Taux d'utilisation des 5 variétés utilisées par les exploitants

\begin{tabular}{|c|c|c|}
\hline Variétés & Cycle & Taux $(\%)$ \\
\hline Gambiaca & 6 Mois & \\
\hline IR15-29 & 6 Mois & $20 \%$ \\
\hline Waihidjo & 5 à 6 Mois & \\
\hline $\mathbf{N}^{\circ} 1$ & 5 à 6 Mois & $80 \%$ \\
\hline $\mathbf{N}^{\circ} \mathbf{2}$ & 5 à 6 Mois & \\
\hline
\end{tabular}

Source : Résultats de l'enquête, 2021

Le tableau 3 montre que les producteurs enquêtés affirment une prédominance de $80 \%$ de la variété locale d'une part d'autre part $20 \%$ des personnes enquêtées attestent que l'adoption des variétés homologuées explique la résilience des cultivateurs exposés au changement climatique. Le tableau 4 indique les facteurs de choix qui contribuent à leur résilience aux changements climatiques. 
Tableau 4 : Efficacité technique d'adoption des variétés

\begin{tabular}{|c|c|c|}
\hline Variétés & Caractéristiques & Horizon temporel \\
\hline Gambiaca & \multirow{2}{*}{$\begin{array}{l}\text { Produit beaucoup et résiste au caprice du climat, talle } \\
\text { bien, très bon goût, résiste bien au stress hydrique }\end{array}$} & \multirow{2}{*}{$\begin{array}{l}\text { Adaptation anticipative à } \\
\text { moyen/ long terme }\end{array}$} \\
\hline IR15-29 & & \\
\hline Waihidjo & \multirow{3}{*}{$\begin{array}{l}\text { Présente les mêmes caractéristiques, résiste au stress } \\
\text { hydrique, mais elle présente une variation de } \\
\text { production en fonction des saisons. }\end{array}$} & \multirow{3}{*}{$\begin{array}{l}\text { Adaptation anticipative à } \\
\text { court terme }\end{array}$} \\
\hline $\mathbf{N}^{\circ} \mathbf{1}$ & & \\
\hline $\mathbf{N}^{\circ} \mathbf{2}$ & & \\
\hline
\end{tabular}

Source : Résultats de l'enquête, 2021

La lecture du tableau 4 montre que les variétés ne sont pas choisies en fonction de la durée de leur cycle, mais beaucoup plus à cause de leur résistance et leur rendement en cas de perturbation des campagnes.

\subsubsection{Déterminants des limites stratégiques d'adaptation}

En réponse aux variabilités climatiques dans la zone d'étude, les exploitants ont développé diverses stratégies classées selon leur appréciation (Tableau 5). Les principaux déterminants de l'adoption des stratégies adaptatives sont variables de perception.

Tableau 5. Limites d'adaptation aux stratégies adoptées

\begin{tabular}{|c|c|c|c|}
\hline Stratégies adaptatives & Degré de prévoyance & Limite d'adaptation & $\operatorname{Taux}(\%)$ \\
\hline $\begin{array}{c}\begin{array}{c}\text { Variétés résistantes au } \\
\text { stress thermique }\end{array} \\
\begin{array}{c}\text { Utilisation de variétés de } \\
\text { cycle court }\end{array}\end{array}$ & $\begin{array}{c}\text { Adaptation spontanée ou } \\
\text { autonome }\end{array}$ & $\begin{array}{l}\text { Accès à l'information et } \\
\text { technique }\end{array}$ & $65 \%$ \\
\hline $\begin{array}{c}\begin{array}{c}\text { l'irrigation de } \\
\text { complément }\end{array} \\
\begin{array}{c}\text { Le drainage et le curage } \\
\text { des cours d'eau }\end{array} \\
\end{array}$ & Adaptation planifiée & Matérielle et techniques & $30 \%$ \\
\hline $\begin{array}{l}\text { Décalage des dates de } \\
\text { semis et irrigation }\end{array}$ & Adaptation anticipative & Techniques et financières & $15 \%$ \\
\hline
\end{tabular}

Source : Résultats de l'enquête, 2021

Il ressort de l'analyse des données du tableau 5 que l'adaptation est limitée par un accès à l'information et technique (65\%) ; Matérielle et techniques $(30 \%)$; Techniques et financières (15\%). Tous ces facteurs peuvent expliquer la faiblesse des exploitants. De ce fait, les financements de l'activité de production du riz constituent la principale limite d'origine des mesures stratégiques. 


\subsection{Facteurs de vulnérabilité}

Le tableau 6 montre que la vulnérabilité des exploitants est multiple et variée

Tableau 6 : Opinions des riziculteurs quant à leur vulnérabilité

\begin{tabular}{|c|c|c|}
\hline Vulnérabilité & Facteurs & Taux (\%) \\
\hline Production & $\begin{array}{l}\text { - retard de semis des cultures } \\
\text { - l'excès de précipitations ou des pluies en } \\
\text { campagne pendant la saison hivernage qui } \\
\text { provoque la prolifération (Parasites des } \\
\text { plantes, les ravageurs et l'érosion du sol) } \\
\text {-Variation de la température au cours de la } \\
\text { période de germination de la semence, de sa } \\
\text { croissance jusqu'à sa récolte (Perte de } \\
\text { rendement) }\end{array}$ & $10 \%$ \\
\hline Financière & $\begin{array}{l}\text { - difficultés d'accéder aux crédits pour -- } \\
\text { l'achat des divers intrants agricoles (les } \\
\text { engrais, les produits phytosanitaires, etc.) } \\
\text {-variation des taux d'intérêt/de la valeur des } \\
\text { actifs financiers/accès au crédit } \\
\text {-l'indisponibilité de la main d'oeuvre }\end{array}$ & $20 \%$ \\
\hline Sociale & $\begin{array}{l}\text {-rupture de la relation entre les exploitants } \\
\text {-les problèmes de santé, } \\
\text {-la perte de main-d'œuvre }\end{array}$ & $70 \%$ \\
\hline
\end{tabular}

Source : Résultats de l'enquête, 2021

Il ressort du tableau 6 que la production ne constitue pas un facteur majeur de leur vulnérabilité. Son indice de vulnérabilité moyen est de $10 \%$. Par contre, le facteur social présente une grande vulnérabilité avec une moyenne de $70 \%$. Ainsi, le comportement social est un facteur de vulnérabilité très inquiétant pour l'avenir.

\section{Discussion}

Les résultats obtenus de cette recherche montrent que les exploitants perçoivent clairement les changements qui interviennent dans leur pratique ainsi que les facteurs qui en sont à l'origine. Cette perception varie selon le niveau d'instruction. L'expérience pratique acquise par ces exploitants sur le terrain est importante, elle n'est cependant complétée par des bonnes pratiques agricoles à travers des associations ou groupements d'intérêt économique, par l'intervention des structures d'encadrement. En effet, le niveau d'instruction des exploitants reste faible à $66 \%$ avec une année d'expérience importante de 10 à 20 ans dans la pratique de la riziculture. Cette corrélation s'explique par le niveau de qualification dans les tâches culturales des acteurs du secteur et aussi une meilleure allocation de la main-d'œuvre. Cette corrélation positive et significative est également obtenue par Kouakou (2017) qui montre que les agriculteurs urbains sont plus souvent spécialisés et bénéficient d'une longue année d'expérience qui permet d'acquérir des aptitudes techniques 
appropriées pour les rendre beaucoup plus utiles. Les résultats de cette recherche révèlent que $90 \%$ des exploitants confirment une augmentation de la température moyenne qui accroit le rendement agricole et d'autre part une conséquence sur les variétés cultivées et une baisse notable du rendement en saison sèche. Ces mêmes observations ont été rapportées par Mallo (2019), pour qui la température constitue le facteur climatique le plus important en ce sens qu'elle est très difficile à modifier. D'après ce dernier, la température optimale pour le développement du riz se situe entre 30 et $35^{\circ} \mathrm{C}$. Par ailleurs, $90 \%$ des exploitants interrogés constatent une augmentation de la pluviométrie avec la variation du niveau du fleuve provoquant la disparition de plusieurs parcelles d'exploitation suite aux inondations qui interviennent généralement en milieu de Campagne Saison Humide. Ces résultats corroborent avec ceux trouvés par Ouédraogo (2012) au Burkina Faso et Garba Hima (2015), toute augmentation des précipitations pourrait être appréhendée comme un excès d'eau pour cette culture et donc aura un effet négatif sur son rendement. Ces résultats montrent que le changement climatique a un effet négatif sur la riziculture irriguée d'une manière ou d'une autre.

Conscients de la réalité du changement climatique, les stratégies développées par les sont pour la plupart anticipatives ou réactives. On distingue l'utilisation des variétés locales, l'utilisation des variétés résistantes, l'irrigation de complément le ressemis et l'utilisation des variétés de cycle court. Ce choix se justifie selon $90 \%$ des riziculteurs comme une alternative et même un palliatif pour sauver la campagne en cas d'urgence. Ces exploitants âgés pratiquent plus des variétés de cycle court sujettes à plus d'impact négatif. En abandonnant la culture du riz, ces exploitants n'ont d'autres alternatives que de vivre à partir des transferts d'argent réalisés par la famille en ville en complément de la pratique de culture moins risquée. Ces résultats sont conformes à ceux identifiés par Naswem et al. (2016) et Arimi, (2014), concernant l'utilisation des variétés améliorées; la culture de variétés résistantes à la sécheresse (Onyegbula et Oladeji, 2017 ; Idoma, et al., 2017), la culture de variétés résistantes à l'inondation (Onyegbula et Oladeji, 2017). Onyegbula et Oladeji (2017) ont souligné le rôle important de l'utilisation des variétés résistantes aux parasites et aux maladies comme une stratégie d'adaptation des riziculteurs au changement climatique. Comme rapporté par Gomgnimbou et al., (2020), ces mêmes stratégies ont été développées par les riziculteurs de la Côte d'Ivoire dans le contexte de changement climatique. Dans le même ordre d'idées, les mêmes constats ont été rapportés par Mossi et al., (2017) sur les périmètres de Tiaguiréré amont, Tiaguiréré aval, Say 1, Say extension, Lata et Daïbéri au Niger où une majorité de producteurs ont opté pour la variété Alewa qui n'est pas homologuée. Plusieurs raisons expliquent le choix porté sur les variétés locales selon les riziculteurs à savoir l'avantage lié à la digestibilité, l'utilité pour le traitement de certaines maladies 
ou au bien-être qu'elles procurent. En effet, il a été rapporté par Gnacadja et al., (2017) que les consommateurs ont plus tendance à avoir une préférence pour le riz produit localement et de qualité, comparés au riz importé. Les résultats de plusieurs auteurs, notamment ceux de Agwata et al., (2014); Tiwari et al., (2014) ; et Obayelu et al., (2014), confirment à ce sujet que l'adoption des techniques d'adaptation et d'atténuation au risque climatique permet d'améliorer les moyens de subsistance et de réduire la vulnérabilité. Cependant, malgré les stratégies mises en œuvre les exploitants enquêtés reconnaissent que ces stratégies comportent certaines contraintes et limites en même temps qu'elles manquent d'efficacité sont eux-mêmes une source de vulnérabilité à la profitabilité par le comportement social qui est un aspect très inquiétant (l'indiscipline dans le respect des règles, querelles, vole). Comme rapporté par Kabore et al., (2019), la variable sexe influence négativement la pratique de l'irrigation (au seuil de $5 \%$ ) dans la mesure où les hommes sont plus impliqués que les femmes dans cette activité et ont plus accès à la terre. De même pour Barry, (2016) ; Opiyo et al., (2016), les caractéristiques socioéconomiques des ménages influencent l'adaptation par leur environnement physique et institutionnel. Les contraintes sociales présentent de nombreuses répercussions vis-à-vis des exploitants et leur degré d'impact dépend notamment les dommages et pertes de la production en l'absence d'une gestion préétablie. De plus, la faible maîtrise de l'itinéraire technique de production sont aussi visibles associée au manque de moyens financier et technique constitue de ce fait les maillons faibles de la production du riz pour accroître les rendements.

\section{Conclusion}

L'adoption d'une stratégie d'adaptation par un exploitant dépend de sa perception du changement climatique et de ses causes, ses impacts négatifs sur l'environnement et des moyens disponibles pour apporter des solutions. Cette étude s'est base sur la perception paysanne des aléas climatiques sur la production du riz dans la vallée du fleuve Niger. Les résultats de la recherche montrent que les exploitants perçoivent clairement les changements qui interviennent dans leur pratique dont la majeure partie des exploitants, le nombre d'actifs ont un niveau d'instruction assez bas, ce qui influence négativement la perception des riziculteurs dans les pratiques collectives. Face à ce nouveau défi, les riziculteurs ont ainsi développé des mesures d'adaptation qui vont de l'adoption des variétés locales à la pratique d'irrigation de complément, l'adaptation des variétés résistantes au stress thermique, le ressemis et l'utilisation des variétés de cycle court. Ces stratégies ne sont qu'une réaction limitée de la part des exploitants face aux effets de la variabilité climatique et manque d'efficacité. La nature et l'efficacité des stratégies utilisées mettent en évidence le caractère vulnérable des exploitants. 
Ainsi, l'analyse des mesures d'adaptation adoptées par les exploitants montre qu'elles sont globalement pertinentes même si ces stratégies sont peu efficaces, elles témoignent néanmoins de la capacité d'adaptation des exploitants malgré leurs limites. En somme, le recours à ces mesures montre que la notion des changements et de variabilité climatiques n'est pas inconnue. Ces derniers sont conscients des risques que représente la variabilité climatique sur leur production et pour leur survie

\section{Remerciements}

Au terme de la présente recherche, nos remerciements vont à l'endroit de tous ceux qui ont contribué pour l'atteinte de ses objectifs. Les auteurs remercient particulièrement le Directeur du périmètre de Saga et les différents Chefs des Groupements Mutualistes de Production (GMP) à travers leur détermination et leur sens de responsabilité, ont fait preuve de collaboration.

\section{References:}

1. Abdou, R., Malice, M., Bakasso, Y., Saadou, M., et Baudoin, J.P., (2014). Taxonomie locale et analyse des critères des paysans pour caractériser les différents écotypes d'oignons (Alliumcepa L.) du Niger. Cah Agric 23: 166-76. Doi: 10.1684/agr.2014.0700

2. Ali, A., et Erenstein, O. (2017). Climate Risk Management assessing farmer use of climate change adaptation practices and impacts on food security and Poverty in Pakistan. Climate Risk Management, 16: 183194.

3. Abid, M., Scheffran, J., Schneider, U. A., et Ashfaq, M.(2015)Farmers' perceptions of and adaptation strategies to climate change and their determinants : the case of Punjab province, Pakistan, Earth Syst. Dynam., 6 : 225-243.DOI : https://doi.org/10.5194/esd-6-2252015

4. Agwata, J., Balew, S., et Anyango, S. (2014). Determinants of adoption choices of climate change adaptation strategies in crop production by small scale farmers in some regions of central Ethiopia. Journal of Natural Science Research, 4 (4): 78-93.

5. Arimi, K. (2014). Determinants of climate change adaptation strategies used by rice farmers in South western, Nigeria. Journal of Agriculture and Rural Development in the Tropics and Subtropics, 115(2): 91-99.

6. Asayehegn, K., Temple, L., Sanchez, B., et Iglesias, A.(2017).Perception of climate change and farm level adaptation choices in central Kenya, Cahiers Agricultures, 26: 25003. DOI : $10.1051 /$ cagri/2017007

7. Bahari, I.M., Ibrahim, B.M., et Oumarou, F.M. (2018). Évolution des caractéristiques pluviométriques et recrudescence des inondations 
dans les localités riveraines du fleuve Niger, Vertigo-la revue électronique en sciences de l'environnement [En ligne], Regards/mis en ligne le 25 mai 2015, consulté le 03 décembre 2021 .URL: http//journal.openedition.org/vertigo/19891; http://doi.org/10.4000/vertigo.19891

8. Barry, S. (2016). Déterminants socioéconomiques et institutionnels de l'adoption des variétés améliorées de maïs dans la région du Centresud du Burkina Faso, Revue d'Economie Théorique et Appliquée, 6(2) : 221-238.

9. Bele, M., Sonwa,D., et Tiani, A., (2014).Local Communities Vulnerability to Climate Change and Adaptation Strategies in Bukavu in DR Congo, Journal of Environment \& Development, (23)3:331357.DOI : 10.1177/1070496514536395

10. Bonkoungou, J., Compaoré, J., et Traore, F. (2019). Analyse de vulnérabilités des systèmes agraires de la région de la Boucle du Mouhoun au Burkina Faso, European Scientific Journal, 15 (2) :104120. URL:http://dx.doi.org/10.19044/esj.2019.v15n2p104

11. Donkora, K. (2019). Évaluation des performances techniques de l'irrigation au Burkina Faso. Thèse de doctorat en sciences agronomiques et ingénierie biologique, Université de Liège Gembloux Agro - Bio Tech, $166 \mathrm{p}$.

12. Dosssouhoui, V.F. (2019). Développement d'un secteur semencier intégré aux chaînes de valeur du riz local au Bénin. Thèse de doctorat $(\mathrm{PhD})$. Université de Liège, Gembloux Agro-Bio Tech (Belgique), 206p.

13. Garba Hima, M.B., et Maman Nafiou, M.M. (2015). Une analyse ricardienne de l'impact de la variabilité de la température et des précipitations sur l'agriculture dans les régions de Dosso et Maradi en République du Niger. Sciences agronomiques, 06 :724-733.

14. Gnacadja, C., Azokpota, P., Moreira, J., et Sie, M. (2017). Perceptions des producteurs et consommateurs sur le riz africain (Oryza glaberrima), Int. J. Biol. Chem. Sci. 11(6) : 2778-2792.

15. Gomgnimbou, A.P.K., Sanon, A.L., Bandaogo, A.A.L., Batieno, A.L., et Nacro, H.B. (2020). Perceptions paysannes du changement climatique et strategies d'adaptation en riziculture pluviale de bas fond dans la région du plateau central du Burkina Faso. J. Rech. Sci. Univ. Lomé (Togo), 22(1) : 23-36.

16. Harvey, C., Rakotobe, Z., Rao, N., Dave, R., Razafimahatratra, R., Rabarijohn, R., Rajaofara, H., et MacKinnon, J.L. (2014). Extreme vulnerability of smallholder farmers to agricultural risks and climate change in Madagascar, Philosophical transactions of royal society, 369:2-22. DOI : http://dx.doi.org/10.1098/rstb.2013.0089. 
17. Idoma, K., Ikpe, E., Ejeh, L., et Mamman, M. (2017). Farmers Adaptation Strategies to the Effect of Climate Variation on Rice Production: Insight from Benue State, Nigeria. Environment and Ecology Research 5(4): 289-301, 2017. DOI: 10.13189/eer.2017.050406

18. Issiaka, Y., Hassane Hamani, S., Abdourahamane Illiassou, S., et Awaiss, A. (2018). Distribution et diversité de la faune aviaire de la ville de Niamey et de sa principale zone humide, le fleuve Niger, Int. J. Biol. Chem. Sci. 12(6) : 2626-2643.

19. Kabore, PN., Barbier, B., Ouoba, P., Kiema, A., Some, L., et Ouédraogo, A. (2019). "Perceptions du changement climatique, impacts environnementaux et stratégies endogènes d'adaptation par les producteurs du Centre-nord du Burkina Faso », VertigO - la revue électronique en sciences de l'environnement [En ligne], Volume 19 Numéro 1 | mars 2019, mis en ligne le 05 mars 2019, consulté le 05 juillet 2021. URL : http://journals.openedition.org/vertigo/24637 ; DOI : $10.4000 /$ vertigo. 24637

20. Kasongo, N., et Mosombo,N. (2017).Perception paysanne des impacts de la variabilité climatique autour de la station de l'INERA/Kipopo dans la province du Katanga en République Démocratique Congo, VertigO - la revue électronique en sciences de l'environnement [En ligne], Volume 17 numéro 3 | décembre 2017, URL : http://journals.openedition.org/vertigo/18873 ;DOI : https://doi .org/10.4000/vertigo. 18873

21. Kouakou, K.P.A. (2017). Analyse de la performance productive de l'agriculture urbaine dans le district d'Abidjan. European Scientific Journal, 13(35) : 288-301.

22. Kosmowski, F., Lalou, R., Sultan, B., Ndiaye, O., Muller, B., Galle, S., et Seguis, L. (2015). Observations et perceptions des changements climatiques: Analyse comparée dans trois pays d'Afrique de l'Ouest. In Sultan B., Lalou R., Sanni M.A. (Editeurs), Les sociétés rurales face aux changements climatiques et environnementaux en Afrique de l'Ouest, 89-110 pp.

23. Mahamadou, I., (2012). La dynamique accélérée des koris dans la région de Niamey : conséquences sur l'ensablement du fleuve Niger, These de doctorat, Université de Niamey, 311p.

24. Kasongo, N., et Mosombo, N.(2017).Perception paysanne des impacts de la variabilité climatique autour de la station de l'INERA/Kipopo dans la province du Katanga en République Démocratique Congo, VertigO - la revue électronique en sciences de l'environnement [En ligne], Volume 17 numéro 3 | décembre 2017, 
URL : http://journals.openedition.org/vertigo/18873 ;DOI : https://doi .org/10.4000/vertigo. 18873

25. Makate, C., Rongchang, W., Makate., et Mango, N., (2016). Crop diversification and livelihoods of smallholder farmers in Zimbabwe: adaptive management for environmental change. Springer Plus 5 : 1135. DOI : 10.1186/s40064-016-2802-4.

26. Mballo, R. (2019). Les communautés adventices du riz irrigué dans la vallée du fleuve Sénégal : structure de la flore, amplitude d'habitat et degrés d'infestation des espèces et amélioration de la gestion de l'enherbement, Thèse de doctorat malherbologie, $161 \mathrm{p}$.

27. Mossi Maïga, I., Alou Himadou, A., Haougui, A., Souleymane, A., et Ibro, G. (2017). Normes techniques et pratiques locales des producteurs dans les perimetres rizicoles irrigués de Toula et de bonfeba au Niger, African crop science journal, 25(4) : 441 - 456.

28. Naswem, A.A., Akpehe, G.A, Awuaga, M.N. (2016). Adaptation Strategies to Climate Change among Rice Farmers in Katsina-Ala Local Government Area of Benue State, Nigeria. Journal of Agriculture and Vétérinairy Science, 9(10): 33-37.

29. Obayelu, O.A., Adepoju, A.O., et Idowu, T. (2014). Factors influencing farmers' choices of adaptation to climate change in Ekiti State, Nigeria. Journal of Agriculture and Environment for International Development, 108 (1): 3-16.

30. Onyegbula, C.B., et Oladeji, J.O. (2017). Utilization of climate change adaptation strategies among rice farmers in three states of Nigeria. Journal of Agricultural Extension and Rural Development. 9 (10): 223229.DOI: 10.5897/JAERD2017.0895

31. Opiyo, F., Wasonga, O.V., Nyangito, M.M., Mureithi, S.M., Obando, J., et Munang, R. (2016). Determinants of perceptions of climate change and adaptation among Turkana pastoralists in Northwestern Kenya, Climate and Developement, 8: 179-189.

32. Ouédraogo, M. (2012). Impact des changements climatiques sur les revenus agricoles au Burkina Faso, Journal of Agriculture and Environment for International Development, 106 (1) : 3-21.

33. Ozer, P., Laminou Manzo, O., Tidjani, A.D., Djaby, B., et DE Longueville. (2017). Evolution récente des extrêmes pluviométriques au Niger (1950-2014). Géo-Eco-Trop., (41)3 : 375-384

34. Pierre, V., Vissoh, R.C., Tossou, H.D., Guibert, H., Olivier, C., Codjia, S., Vodouhe, D., et Agbossou, K.E. (2012). Perceptions et stratégies d'adaptation aux changements climatiques : le cas des communes d'Adjohoun et de Dangbo au Sud-Est Bénin, Les Cahiers d'Outre-Mer [En ligne], 260 | Octobre-Décembre 2012, mis en ligne le 01 octobre 
2015, consulté le 03 Juillet 2021. URL : http://journals.openedition.org/com/6700 ; DOI : 10.4000/com.6700

35. Saboury, N.M. (2018) Impact de l'adoption des stratégies d'adaptation au changement climatique sur la sécurité alimentaire et le revenu des ménages pastoraux dans la zone du Ferlo au Sénégal, thèse de doctorat en économie Agricole et Sociologie, Université de Thiès au Sénégal, $184 \mathrm{p}$.

36. Sarr, B., Atta, S., Ly, M., Salack, S., Ourback, T., Subsol, S., et George., (2015). Adaptating to climate variability and change in smallholder farming communities : a case study from Burkina-Faso, chad and Niger, Journal of agricultural extension and rural development , 1 : 418-429.

37. Tiwari, K.R., Rayamajhi, S., Pokharel, R.K., et Balla, M.K. (2014), Determinants of the climate change adaptation in rural Farming in Nepal Himalaya. International journal of multidisciplinary and current Research, 2 : 234-240.

38. Yegbemey, RN., Yabi, J.A., Aïhounton, G.B., et Paraison, A. (2014). Modélisation simultanée de la perception et de l'adaptation au changement climatique : cas des producteurs de maïs du Nord Benin. Cahier de l'agriculture ,23:177-188. 